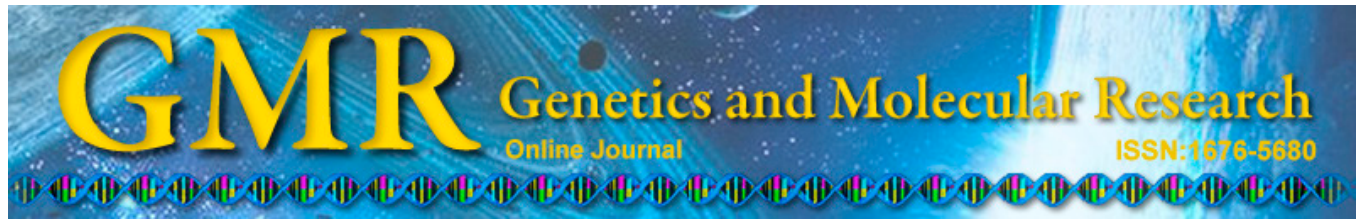

\title{
Estrogen receptor 1 PvuII and XbaI polymorphisms and susceptibility to Alzheimer's disease: a meta-analysis
}

\author{
Y.H. Lee and G.G. Song \\ Division of Rheumatology, Department of Internal Medicine, \\ Korea University College of Medicine, Seoul, Korea \\ Corresponding author: Y.H. Lee \\ E-mail: lyhcgh@korea.ac.kr \\ Genet. Mol. Res. 14 (3): 9361-9369 (2015) \\ Received January 14, 2015 \\ Accepted March 23, 2015 \\ Published August 10, 2015 \\ DOI http://dx.doi.org/10.4238/2015.August.10.17
}

\begin{abstract}
The aim of this study was to explore whether estrogen receptor 1 (ESR1) PvuII and $X b a \mathrm{I}$ polymorphisms are associated with susceptibility to Alzheimer's disease (AD). We conducted a metaanalysis of the associations between AD and ESR1 PvuII and XbaI polymorphisms as well as haplotypes of the ESR1 PvuII and XbaI polymorphisms. A total of 1359 patients and 1387 controls from 9 studies on the ESR1 PvuII polymorphism and 1525 patients and 1575 controls from 8 studies on the ESR1 XbaI polymorphism were included in this meta-analysis. Gender-specific meta-analysis showed an association between the ESR $1 P P+P p$ genotype and AD in males $(\mathrm{OR}=0.302,95 \% \mathrm{CI}=0.100-0.914, \mathrm{P}=0.034)$, but not in females. No association was observed between $\mathrm{AD}$ and the ESR $1 \mathrm{Xba \textrm {I } X}$ allele (OR $=1.114,95 \% \mathrm{CI}=0.868-1.429, \mathrm{P}=0.397)$. However, country-specific meta-analysis identified an association between AD and the ESR $1 X$ allele in Japanese $(\mathrm{OR}=1.386,95 \% \mathrm{CI}=1.055-1.822, \mathrm{P}=0.019)$, but not Chinese or Italian populations. Meta-analyses results indicated an association between the $\mathrm{PP} / \mathrm{XX}$ haplotypes and $\mathrm{AD}$ in Chinese
\end{abstract}


population $(\mathrm{OR}$ for $\mathrm{PP} / \mathrm{XX} v s$ others $=2.758,95 \% \mathrm{CI}=1.750-4.346$, $\left.\mathrm{P}=1.2 \times 10^{-6}\right)$. This meta-analysis showed associations between the ESRI $P v u I I$ polymorphism and AD susceptibility in males, between $\mathrm{AD}$ risk and the ESRI XbaI polymorphism in the Japanese population, and between the $\mathrm{PP} / \mathrm{XX}$ haplotype and AD susceptibility in the Chinese population.

Key words: Alzheimer's disease; Estrogen receptor; Polymorphism; Meta-analysis

\section{INTRODUCTION}

Alzheimer's disease (AD) is a complex, progressive, and irreversible neurodegenerative disease of the brain. $\mathrm{AD}$ is characterized by the accumulation of the amyloid- $\beta$ (A $\beta$ ) protein and neurofibrillary tangles in the brain (Boada et al., 2012a). Although the etiology of AD has not been determined, a genetic component of susceptibility to AD has been established by some case-control, twin, and family studies.

Estrogen induces synaptogenesis, acts as a neuroprotective agent, regulates neurotransmission in the central nervous system (CNS), and is associated with cognitive symptoms (Szego et al., 2011). Estrogen may inhibit the formation of the $\mathrm{A} \beta$ protein and protect against A $\beta$ protein-induced neuronal death (Szego et al., 2011), however AD may be more prevalent in women, attributed in part, to estrogen (Bachman et al., 1992). Thus estrogen is considered to play a role in the pathogenesis of AD (Xing et al., 2013). Estrogen action is mediated through estrogen receptors 1 (ESR1) and 2 (ESR2). The ESR1 gene, located on chromosome $6 \mathrm{q} 25.1$, is expressed in the brain, predominantly in the hypothalamus and amygdale (Xing et al., 2013). ESR1 polymorphisms located in intron 1, PvuII (rs2234693) and XbaI (rs9340799), may be in linkage disequilibrium (LD) with regulatory polymorphisms that affect ESR1 expression or disease susceptibility genes. The ESR1 PvuII and XbaI polymorphisms have been the most intensively studied in association with susceptibility to AD and whether ESRlgene plays a role in the pathogenesis of AD (Xing et al., 2013).

Published results on the genetic association of ESR1 polymorphisms are controversial and inconclusive. While some reports have shown associations between these ESR1 polymorphisms and AD, others found no such association (Ji et al., 2000; Maruyama et al., 2000; Lambert et al., 2001; Lin et al., 2003; Monastero et al., 2006; Porrello et al., 2006; Usui et al., 2006; Dresner-Pollak et al., 2009; Ma et al., 2009; Boada et al., 2012b; Deng et al., 2013). This discrepancy may be due to sample size, statistical power, ethnicity, gender, clinical heterogeneity, or a combination of these factors. Meta-analysis is a powerful tool to overcome the drawbacks of small sample size and inadequate statistical power from genetic studies of complex traits. To overcome the limitations of individual studies, we carried out a meta-analysis (Lee and Nath, 2005; Lee et al., 2005, 2006) to determine whether the ESR1 PvuII and XbaI polymorphisms are associated with susceptibility to AD.

\section{METHODS}

\section{Identification of eligible studies and data extraction}

Using MEDLINE and EMBASE citation databases, we performed a literature search 
to identify articles published up to October 2013 that examined associations between ESRI polymorphisms and AD. Combinations of key words such as "estrogen receptor 1", "ESRl", "polymorphism", "Alzheimer's disease", and "AD" were entered as Medical Subject Heading terms and text words. References in the studies identified were used to identify additional studies not indexed by the electronic databases. Inclusion criteria were as follows: 1) case-control study design, 2) original data, 3) genotype or allele data to calculate odds ratios (ORs), and 4) studies in which the genotype distribution in the controls was not consistent with the HardyWeinberg equilibrium (HWE), because such deviations in HWE among controls suggests the possibility of bias during control selection or genotyping errors. The following information was extracted from each study identified: author, year of publication, ethnicity and country of the study population, demographics, and number of cases and controls for each ESR1 polymorphism. Allele frequencies were calculated from the corresponding genotype distributions.

\section{Evaluation of statistical associations}

A chi-square test was used to determine whether observed genotype frequencies conformed to HWE. Point estimates of risks, ORs, and 95\% confidence intervals (CIs) were estimated for each study. Cochran's Q-statistic was used to assess intra- and inter-study variation or heterogeneity. $\mathrm{I}^{2}$ values ranged between 0 and $100 \%$, representing the proportion of interstudy variability attributable to heterogeneity rather than chance (Higgins and Thompson, 2002). $I^{2}$ values of 25,50 , and $75 \%$ were nominally assigned as low, moderate, and high estimates, respectively. The fixed-effects model assumed that a genetic factor had the same effect on AD susceptibility across all studies investigated and that variations between studies were caused by chance alone. The random-effects model assumed that different studies had substantial diversity and assessed intra-study sampling error and inter-study variance (DerSimonian and Laird, 1986). A comprehensive meta-analysis program (Biostat Englewood, NJ, USA) was used for analysis. Study power was computed as the probability of detecting an association between the ESR1 polymorphisms and AD at a significance level of 0.05 , assuming an OR of 1.5 (small effect size). Power analysis was performed using the G*Power statistical program (http://www.psycho.uni-duesseldorf.de/aap/projects/gpower).

\section{Evaluation of heterogeneity and publication bias}

Meta-regression was performed to examine the potential source of heterogeneity observed in the meta-analysis. Sensitivity analysis was also performed to assess the influence of each individual study on the pooled OR by omitting each individual study. We evaluated publication bias using Egger's linear regression test, which measures funnel plot asymmetry using a natural logarithmic scale of ORs. When asymmetry was indicated, we used the trim and fill method to adjust the summary estimate for the bias observed.

\section{RESULTS}

\section{Studies included in the meta-analysis}

We identified 135 studies by electronic and manual search, of which 16 studies were selected for a full-text review on the basis of the title and abstract details (Isoe-Wada et al., 
1999; Ji et al., 2000; Maruyama et al., 2000; Lambert et al., 2001; Lin et al., 2003; den Heijer et al., 2004; Pirskanen et al., 2005; Monastero et al., 2006; Porrello et al., 2006; Usui et al., 2006; Boccardi et al., 2008; Dresner-Pollak et al., 2009; Ma et al., 2009; Goumidi et al., 2011; Boada et al., 2012b; Deng et al., 2013). After full-text review, seven studies were excluded, of which 4 did not contain genotype data for ESR1 polymorphism (den Heijer et al., 2004; Pirskanen et al., 2005; Boccardi et al., 2008; Goumidi et al., 2011), 2 contained data on control genotype deviated from HWE (Maruyama et al., 2000; Boada et al., 2012b) and 1 study contained duplicate data (Isoe-Wada et al., 1999). Thus, a total of 9 studies (Ji et al., 2000; Lambert et al., 2001; Lin et al., 2003; Monastero et al., 2006; Porrello et al., 2006; Usui et al., 2006; Dresner-Pollak et al., 2009; Ma et al., 2009; Deng et al., 2013), corresponding to 1359 patients and 1387 controls, met all the inclusion criteria and were considered for the ESR1 XbaI polymorphism meta-analysis (Table 1C). Of these, 8 studies corresponding to 1525 patients and 1575 controls were also included in the ESR1 PvuII polymorphism meta-analysis (Table 1B). The statistical power of the studies ranged from 23.6 to $68.1 \%$ and none had statistical power exceeding $80 \%$.

Table 1. Characteristics of studies used in the meta-analysis.

\begin{tabular}{|c|c|c|c|c|c|c|c|c|c|c|c|c|}
\hline \multirow[t]{2}{*}{$\begin{array}{l}\text { Clinical characteristics } \\
\text { Citation }\end{array}$} & \multirow{2}{*}{\multicolumn{2}{|c|}{ Country Population }} & \multicolumn{4}{|c|}{ Gender (Female, \%) } & \multicolumn{4}{|c|}{ Age at examination (means \pm SD) } & \multicolumn{2}{|c|}{ Onset age (means $\pm \mathrm{SD}$ ) } \\
\hline & & & Case & \multicolumn{3}{|c|}{ Control } & \multicolumn{2}{|c|}{ Case } & \multicolumn{2}{|r|}{ Control } & Case & Control \\
\hline Deng et al., 2013 & China & Clinic & 66.5 & \multicolumn{2}{|r|}{66.5} & & \multicolumn{2}{|c|}{$67.3 \pm 7.2$} & \multicolumn{2}{|r|}{$66.4 \pm 9.1$} & NA & NA \\
\hline Ma et al., 2009 & China & Community & 84.3 & \multicolumn{2}{|r|}{75.5} & \multicolumn{3}{|c|}{$81.4 \pm 6.7$} & \multicolumn{2}{|r|}{$73 \pm 6.3$} & NA & NA \\
\hline Dresner-Pollak et al., 2009 & Israel & Community & NA & \multirow{2}{*}{\multicolumn{2}{|c|}{ NA }} & & \multicolumn{2}{|c|}{$83.5 \pm 7.3$} & \multicolumn{2}{|r|}{$81.0 \pm 7.1$} & NA & NA \\
\hline Monastero et al., 2006 & Italy & Clinic & 65.1 & 65.1 & & & \multicolumn{2}{|c|}{$73.7 \pm 8.0$} & \multicolumn{2}{|r|}{$72.9 \pm 7.9$} & $70.8 \pm 8.0$ & NA \\
\hline Porrello et al., 2006 & Italy & Clinic & 56 & & 53 & & $75 \pm$ & & & $71 \pm 10$ & NA & NA \\
\hline Usui et al., 2006 & Japan & Clinic & 54.1 & & 35.8 & & 69.1 & 10.7 & & 62.89 .0 & NA & NA \\
\hline Lin et al., 2003 & China & Communi & 73.1 & & 50.4 & & 791.4 & & & NA & NA & NA \\
\hline Lambert et al., 2001 & UK & Clinic & 61 & & 53 & & $67.9 \pm$ & 12.5 & & $62.6 \pm 14.9$ & 64.611 .8 & NA \\
\hline Ji et al., 2000 & Japan & Clinic & NA & & NA & & $78.6 \pm$ & & & $75.5 \pm 5.5$ & NA & NA \\
\hline ESRl PvuII polymorphism & & & & & & & & & & & & \\
\hline Citation & Ethnicity & & imbers & & Case & & & Contro & & HWE As & ssociation $\mathrm{P}^{\mathrm{a}}$ & Power $(\%)^{b}$ \\
\hline & & Case & Control & PP & $\mathrm{Pp}$ & $\mathrm{pp}$ & PP & $\mathrm{Pp}$ & $\mathrm{pp}$ & & & \\
\hline Deng et al., 2013 & Asian & 236 & 236 & 46 & 123 & 67 & 53 & 127 & 56 & 0 & 0.241 & 58.4 \\
\hline Ma et al., 2009 & Asian & 219 & 215 & 24 & 113 & 82 & 34 & 110 & 71 & 0 & 0.162 & 54.9 \\
\hline Dresner-Pollak et al., 2009 & Jewish & 118 & 68 & $181^{*}$ & & & $86^{*}$ & & & 0 & 0.006 & 27.5 \\
\hline Porrello et al., 2006 & European & 131 & 109 & 23 & 62 & 46 & 24 & 45 & 40 & 0 & 0.750 & 34.0 \\
\hline Usui et al., 2006 & Asian & 205 & 92 & 43 & 91 & 71 & 19 & 41 & 32 & 0 & 0.957 & 40.6 \\
\hline Lin et al., 2003 & Asian & 30 & 128 & 7 & 17 & 6 & 19 & 56 & 53 & 0 & 0.035 & 34.1 \\
\hline Lambert et al., 2001 & European & 186 & 405 & 36 & 96 & 54 & 77 & 200 & 128 & 0 & 0.639 & 68.1 \\
\hline Ji et al., 2000 & Asian & 234 & 134 & 59 & 114 & 61 & 21 & 61 & 52 & 0 & 0.004 & 48.3 \\
\hline ESR1 XbaI polymorphism & & & & & & & & & & & & \\
\hline Citation & Ethnicity & & imbers & & Case & & & Contro & & HWE As & ssociation $\mathrm{P}^{\mathrm{a}}$ & Power $(\%)^{b}$ \\
\hline & & Case & Control & $\mathrm{XX}$ & $\mathrm{Xx}$ & $\mathrm{xx}$ & $\mathrm{XX}$ & $\mathrm{Xx}$ & $\mathrm{xx}$ & & & \\
\hline Deng et al., 2013 & Asian & 236 & 236 & 22 & 93 & 121 & 35 & 105 & 96 & 0 & 0.009 & 58.4 \\
\hline Ma et al., 2009 & Asian & 228 & 234 & 21 & 84 & 123 & 22 & 99 & 113 & 0 & 0.328 & 57.5 \\
\hline Dresner-Pollak et al., 2009 & Jewish & 118 & 68 & $177^{*}$ & & & $80 *$ & & & 0 & 0.001 & 27.5 \\
\hline Monastero et al., 2006 & European & 158 & 172 & 18 & 92 & 48 & 33 & 98 & 41 & 0 & 0.064 & 44.3 \\
\hline Porrello et al., 2006 & European & 131 & 109 & 15 & 61 & 55 & 14 & 48 & 47 & 0 & 0.976 & 34 \\
\hline Usui et al., 2006 & Asian & 205 & 92 & 18 & 59 & 128 & 2 & 36 & 54 & 0 & 0.700 & 40.6 \\
\hline Lin et al., 2003 & Asian & 29 & 125 & 6 & 9 & 14 & 8 & 32 & 85 & 0 & 0.006 & 23.6 \\
\hline Lambert et al., 2001 & European & 186 & 405 & 21 & 88 & 77 & 43 & 197 & 165 & 0 & 0.998 & 68.1 \\
\hline Ji et al., 2000 & Asian & 234 & 134 & 23 & 90 & 121 & 6 & 41 & 87 & 0 & 0.006 & 48.3 \\
\hline
\end{tabular}

SD, Standard deviation; NA, Not available; UK, United Kingdom; HWE, Hardy-Weinberg equilibrium; 0, control genotype in HWE; ${ }^{2}$ Allelic association; ${ }^{b}$ Assuming an odds ratio of 1.5 (small effect size) at a level of significance of $0.05, *$ Allele number. 


\section{Meta-analysis of the ESR1 PvuII polymorphism and AD}

No association was observed between $\mathrm{AD}$ and the ESRI PvuII P allele in the combined study subjects $(\mathrm{OR}=1.135,95 \% \mathrm{CI}=0.920-1.401, \mathrm{P}=0.238)$ (Table 2, Figure 1$)$. Country-specific meta-analysis indicated no association between the ESRI P allele and $\mathrm{AD}$ in either Chinese (OR $=0.999,95 \% \mathrm{CI}=0.700-1.424, \mathrm{P}=0.993)$ or Japanese $(\mathrm{OR}=1.272,95 \% \mathrm{CI}=0.823-1.966, \mathrm{P}=$ 0.279 ) population (Table 2). ESR1 polymorphism data in both, males and females, was presented in 2 studies (Lambert et al., 2001; Lin et al., 2003). Gender-specific meta-analysis of results from these studies indicated no association between the ESR1 $\mathrm{P}$ allele and $\mathrm{AD}$ in females $(\mathrm{OR}=1.496$, $95 \% \mathrm{CI}=0.724-3.091, \mathrm{P}=0.277)$ or males $(\mathrm{OR}=1.187,95 \% \mathrm{CI}=0.821-1.716, \mathrm{P}=0.363)($ Table 2). However, meta-analysis using dominant model showed an association between the ESRI PvuII polymorphism and $\mathrm{AD}$ in males $(\mathrm{OR}=0.302,95 \% \mathrm{CI}=0.100-0.914, \mathrm{P}=0.034)$, but not in females.

Table 2. Analysis of the association between the ESR1 PvuII polymorphism and AD.

\begin{tabular}{|c|c|c|c|c|c|c|c|c|}
\hline \multirow[t]{2}{*}{ Polymorphism } & \multirow[t]{2}{*}{ Population } & \multirow[t]{2}{*}{ No. of studies } & \multicolumn{3}{|c|}{ Test of association } & \multicolumn{3}{|c|}{ Test of heterogeneity } \\
\hline & & & OR & $95 \% \mathrm{CI}$ & $\mathrm{P}$ & Model & $\mathrm{P}$ & $I^{2}$ \\
\hline \multirow[t]{5}{*}{$\mathrm{P} v s \mathrm{p}$} & Overall & 8 & 1.135 & $0.920-1.401$ & 0.238 & $\mathrm{R}$ & 0.002 & 69.2 \\
\hline & Chinese & 3 & 0.999 & $0.700-1.424$ & 0.993 & $\mathrm{R}$ & 0.035 & 70.5 \\
\hline & Japanese & 2 & 1.272 & $0.823-1.966$ & 0.279 & $\mathrm{R}$ & 0.061 & 71.4 \\
\hline & Female & 2 & 1.496 & $0.724-3.091$ & 0.277 & $\mathrm{R}$ & 0.052 & 73.4 \\
\hline & Male & 2 & 1.187 & $0.821-1.716$ & 0.363 & $\mathrm{~F}$ & 0.340 & 0 \\
\hline \multirow[t]{5}{*}{$\mathrm{p} v s \mathrm{P}$} & Overall & 8 & 0.88 & $0.714-1.087$ & 0.238 & $\mathrm{R}$ & 0.002 & 69.2 \\
\hline & Chinese & 3 & 1.001 & $0.702-1.428$ & 0.993 & $\mathrm{R}$ & 0.035 & 70.5 \\
\hline & Japanese & 2 & 0.786 & $0.509-1.215$ & 0.279 & $\mathrm{R}$ & 0.061 & 71.4 \\
\hline & Female & 2 & 0.669 & $0.324-1.381$ & 0.277 & $\mathrm{R}$ & 0.052 & 73.4 \\
\hline & Male & 2 & 0.843 & $0.583-1.218$ & 0.363 & $\mathrm{~F}$ & 0.340 & 0 \\
\hline \multirow[t]{5}{*}{$\mathrm{PP} v s \mathrm{Pp}+\mathrm{pp}$ (Recessive) } & Overall & 7 & 0.990 & $0.803-1.220$ & 0.923 & $\mathrm{~F}$ & 0.157 & 35.4 \\
\hline & Chinese & 3 & 0.836 & $0.602-1.160$ & 0.283 & $\mathrm{~F}$ & 0.233 & 31.3 \\
\hline & Japanese & 2 & 1.398 & $0.930-2.102$ & 0.108 & $\mathrm{~F}$ & 0.168 & 47.2 \\
\hline & Female & 2 & 1.152 & $0.668-0.987$ & 0.611 & $\mathrm{~F}$ & 0.196 & 40.0 \\
\hline & Male & 2 & 0.704 & $0.389-1.273$ & 0.245 & $\mathrm{~F}$ & 0.617 & 0 \\
\hline \multirow[t]{5}{*}{$\mathrm{PP}+\mathrm{Pp} v s \mathrm{pp}$ (Dominant) } & Overall & 7 & 1.117 & $0.856-1.458$ & 0.416 & $\mathrm{R}$ & 0.044 & 53.6 \\
\hline & Chinese & 3 & 1.024 & 0.603-1.739 & 0.929 & $\mathrm{R}$ & 0.048 & 66.9 \\
\hline & Japanese & 2 & 1.364 & $0.773-2.407$ & 0.284 & $\mathrm{R}$ & 0.098 & 63.4 \\
\hline & Female & 2 & 1.899 & $0.667-5.403$ & 0.229 & $\mathrm{R}$ & 0.076 & 68.2 \\
\hline & Male & 2 & 0.302 & $0.100-0.914$ & 0.034 & $\mathrm{R}$ & 0.061 & 71.5 \\
\hline \multirow[t]{5}{*}{$\mathrm{PP} v s \mathrm{pp}$} & Overall & 7 & 0.928 & $0.729-1.180$ & 0.541 & $\mathrm{~F}$ & 0.100 & 43.5 \\
\hline & Chinese & 3 & 0.781 & $0.541-1.127$ & 0.187 & $\mathrm{~F}$ & 0.421 & 0 \\
\hline & Japanese & 2 & 1.525 & $0.939-2.478$ & 0.088 & $\mathrm{~F}$ & 00175 & 45.6 \\
\hline & Female & 2 & 2.062 & $0.479-8.874$ & 0.331 & $\mathrm{R}$ & 0.072 & 69.0 \\
\hline & Male & 2 & 1.427 & $0.663-3.070$ & 0.363 & $\mathrm{~F}$ & 0.357 & 0 \\
\hline
\end{tabular}

ESR1, estrogen receptor 1; HWE, Hardy-Weinberg equilibrium; F, fixed model; R, random model.

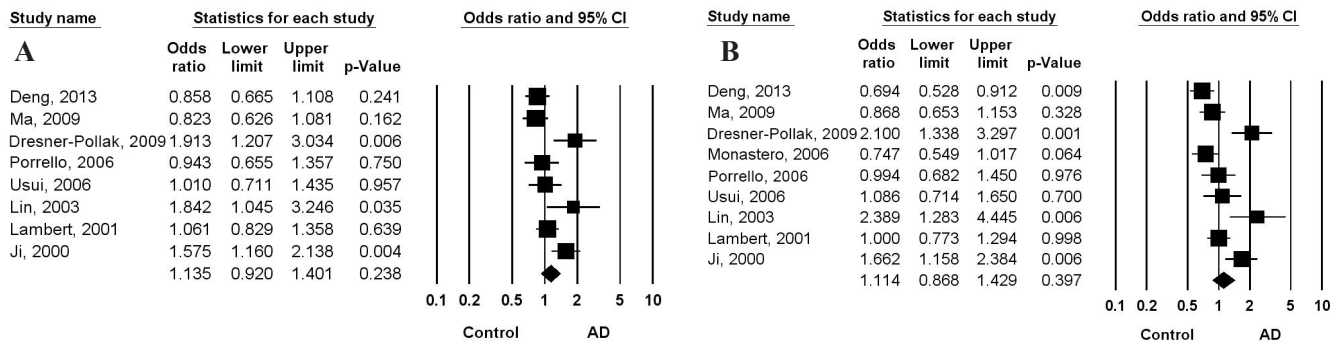

Figure 1. ORs and $95 \%$ CIs of individual studies and pooled data for the allelic association between AD and ESRI $P v u I I(A)$ and $X b a I$ (B) polymorphisms in all subjects. 


\section{Meta-analysis of ESR1 XbaI polymorphism and AD}

Meta-analysis of the combined study subjects showed no association between $\mathrm{AD}$ and the ESR1 XbaI X allele (OR $=1.114,95 \% \mathrm{CI}=0.868-1.429, \mathrm{P}=0.397)$ (Table 3, Figure 2).

However, country-specific meta-analysis identified an association between $\mathrm{AD}$ and the ESR $1 \mathrm{Xba \textrm {I } X}$ allele in Japanese $(\mathrm{OR}=1.386,95 \% \mathrm{CI}=1.055-1.822, \mathrm{P}=0.019)$, but not in either Chinese or Italian population (Table 3). Gender-specific meta-analysis indicated no association between the ESR1 X allele and AD in both, males and females (Table 3).

Table 3. Analysis of the association between $\mathrm{AD}$ and the ESR1 XbaI polymorphism.

\begin{tabular}{|c|c|c|c|c|c|c|c|c|}
\hline \multirow[t]{2}{*}{ Polymorphism } & \multirow[t]{2}{*}{ Population } & \multirow[t]{2}{*}{ No. of studies } & \multicolumn{3}{|c|}{ Test of association } & \multicolumn{3}{|c|}{ Test of heterogeneity } \\
\hline & & & OR & $95 \% \mathrm{CI}$ & $\mathrm{P}$ & Model & $\mathrm{P}$ & $I^{2}$ \\
\hline \multirow[t]{6}{*}{$\mathrm{X} v s \mathrm{X}$} & Overall & 9 & 1.114 & $0.868-1.429$ & 0.397 & $\mathrm{R}$ & 0.000 & 78.3 \\
\hline & Chinese & 3 & 1.040 & $0.618-1.750$ & 0.884 & $\mathrm{R}$ & 0.002 & 84.3 \\
\hline & Japanese & 2 & 1.386 & $1.055-1.822$ & 0.019 & $\mathrm{~F}$ & 0.131 & 56.1 \\
\hline & Italian & 2 & 0.838 & $0.660-1.064$ & 0.147 & $\mathrm{~F}$ & 0.131 & 56.1 \\
\hline & Female & 2 & 1.309 & $0.956-1.792$ & 0.094 & $\mathrm{~F}$ & 0.119 & 58.8 \\
\hline & Male & 2 & 1.780 & $0.554-5.714$ & 0.333 & $\mathrm{R}$ & 0.067 & 71.6 \\
\hline \multirow[t]{6}{*}{$\mathrm{x} v s \mathrm{X}$} & Overall & 9 & 0.898 & $0.700-1.152$ & 0.397 & $\mathrm{R}$ & 0.000 & 78.3 \\
\hline & Chinese & 3 & 0.962 & $0.571-1.619$ & 0.884 & $\mathrm{R}$ & 0.002 & 84.3 \\
\hline & Japanese & 2 & 0.721 & $0.549-0.948$ & 0.019 & $\mathrm{~F}$ & 0.131 & 56.1 \\
\hline & Italian & 2 & 1.193 & $0.940-1.515$ & 0.147 & $\mathrm{~F}$ & 0.131 & 56.1 \\
\hline & Female & 2 & 0.764 & $0.558-1.047$ & 0.094 & $\mathrm{~F}$ & 0.119 & 58.8 \\
\hline & Male & 2 & 0.562 & $0.175-1.804$ & 0.333 & $\mathrm{R}$ & 0.067 & 71.6 \\
\hline \multirow[t]{6}{*}{$\mathrm{XX}$ vs $\mathrm{Xx}+\mathrm{xx}$ (Recessive) } & Overall & 8 & 1.127 & $0.722-1.757$ & 0.599 & $\mathrm{R}$ & 0.007 & 63.77 \\
\hline & Chinese & 3 & 1.138 & $0.479-2.704$ & 0.770 & $\mathrm{R}$ & 0.016 & 75.7 \\
\hline & Japanese & 2 & 2.768 & $1.263-6.067$ & 0.011 & $\mathrm{~F}$ & 0.485 & 0 \\
\hline & Italian & 2 & 0.653 & $0.402-1.061$ & 0.085 & $\mathrm{~F}$ & 0.342 & 0 \\
\hline & Female & 2 & 1.469 & $0.770-2.801$ & 0.243 & $\mathrm{~F}$ & 0.809 & 0 \\
\hline & Male & 2 & 4.525 & $0.352-58.13$ & 0.246 & $\mathrm{R}$ & 0.017 & 82.4 \\
\hline \multirow[t]{6}{*}{$\mathrm{XX}+\mathrm{Xx}$ vs $\mathrm{xx}$ (Dominant) } & Overall & 8 & 0.973 & $0.752-1.258$ & 0.835 & $\mathrm{R}$ & 0.012 & 61.3 \\
\hline & Chinese & 3 & 0.929 & $0.550-1.570$ & 0.784 & $\mathrm{R}$ & 0.024 & 73.2 \\
\hline & Japanese & 2 & 1.229 & $0.617-2.450$ & 0.557 & $\mathrm{R}$ & 0.038 & 76.7 \\
\hline & Italian & 2 & 0.158 & $0.603-1.223$ & 0.397 & $\mathrm{~F}$ & 0.295 & 88.9 \\
\hline & Female & 2 & 1.658 & $0.674-4.081$ & 0.271 & $\mathrm{R}$ & 0.091 & 65.0 \\
\hline & Male & 2 & 1.115 & $0.651-1.908$ & 0.692 & $\mathrm{~F}$ & 0.606 & 0 \\
\hline \multirow[t]{6}{*}{$\mathrm{XX} v s \mathrm{xx}$} & Overall & 8 & 1.130 & $0.675-1.892$ & 0.643 & $\mathrm{R}$ & 0.002 & 69.3 \\
\hline & Chinese & 3 & 1.105 & $0.399-3.064$ & 0.848 & $\mathrm{R}$ & 0.005 & 81.2 \\
\hline & Japanese & 2 & 3.018 & $1.362-6.687$ & 0.007 & $\mathrm{~F}$ & 0.722 & 0 \\
\hline & Italian & 2 & 0.621 & $0.362-1.063$ & 0.082 & $\mathrm{~F}$ & 0.224 & 32.3 \\
\hline & Female & 2 & 1.623 & $0.822-3.025$ & 0.163 & $\mathrm{~F}$ & 0.548 & 0 \\
\hline & Male & 2 & 3.590 & $0.424-30.41$ & 0.241 & $\mathrm{R}$ & 0.048 & 74.4 \\
\hline
\end{tabular}

ESR1, estrogen receptor 1; F, fixed model; R, random model.

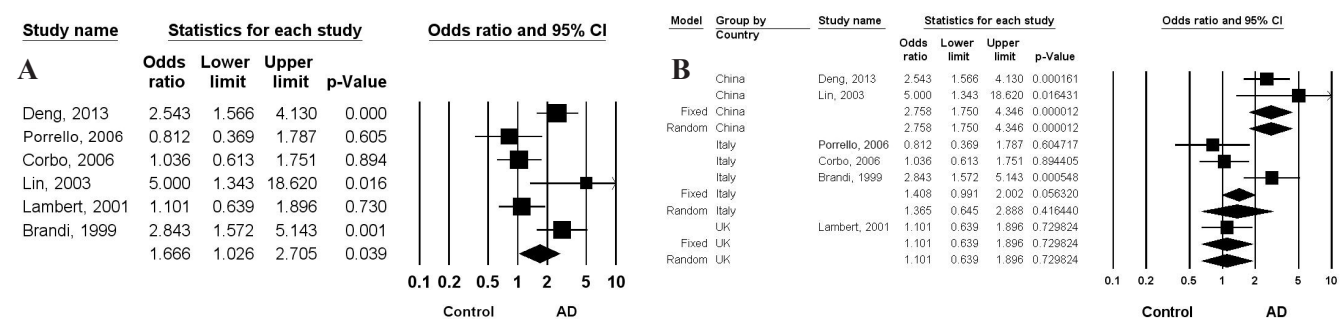

Figure 2. ORs and $95 \% \mathrm{CIs}$ of individual studies and pooled data for the allelic association between $\mathrm{AD}$ and the ESR1 PvuII-XbaI haplotype in all subjects (A) and in each country group (B). 


\section{Meta-analysis of PvuII-XbaI haplotype and AD}

Meta-analyses of the $P v u \mathrm{II}-X b a \mathrm{I}$ haplotype showed a significant association between the $\mathrm{PP} / \mathrm{XX}$ haplotypes and $\mathrm{AD}(\mathrm{OR}$ for $\mathrm{PP} / \mathrm{XX}$ s others $=1.666,95 \% \mathrm{CI}=0.026-2.705, \mathrm{P}=$ 0.039; OR for PP/XX vs pp/xx = 2.071, 95\%CI $=1.150-6.368, \mathrm{P}=0.023$ ) (Table 4). Countryspecific meta-analysis indicated an association between the PP/XX haplotypes and AD Chinese $\left(\mathrm{OR}\right.$ for $\mathrm{PP} / \mathrm{XX} v s$ others $=2.758,95 \% \mathrm{CI}=1.750-4.346, \mathrm{P}=1.2 \times 10^{-6}$; OR for PP/XX $\left.v s \mathrm{pp} / \mathrm{xx}=14.66,95 \% \mathrm{CI}=6.729-31.95, \mathrm{P}<1.0 \times 10^{-8}\right)$, but not in Italian population (Table 4).

\begin{tabular}{|c|c|c|c|c|c|c|c|c|}
\hline \multirow[t]{2}{*}{ Polymorphism } & \multirow[t]{2}{*}{ Population } & \multirow[t]{2}{*}{ No. of studies } & \multicolumn{3}{|c|}{ Test of association } & \multicolumn{3}{|c|}{ Test of heterogeneity } \\
\hline & & & OR & $95 \% \mathrm{CI}$ & $\mathrm{P}$ & Model & $\mathrm{P}$ & $I^{2}$ \\
\hline \multirow[t]{5}{*}{$\mathrm{PP} / \mathrm{XX} v s$ others } & Overall & 6 & 1.666 & $1.026-2.705$ & 0.039 & $\mathrm{R}$ & 0.004 & 71.6 \\
\hline & Chinese & 2 & 2.758 & $1.750-4.346$ & $1.2 \times 10^{-6}$ & $\mathrm{~F}$ & 0.344 & 0 \\
\hline & Italian & 3 & 1.365 & $0.645-2.888$ & 0.416 & $\mathrm{R}$ & 0.014 & 76.7 \\
\hline & Female & 2 & 1.526 & $0.797-2.924$ & 0.203 & $\mathrm{~F}$ & 0.613 & 0 \\
\hline & Male & 2 & 5.569 & $0.349-88.90$ & 0.224 & $\mathrm{R}$ & 0.043 & 75.5 \\
\hline \multirow[t]{5}{*}{$\mathrm{PP} / \mathrm{XX} v s \mathrm{pp} / \mathrm{xx}$} & Overall & 6 & 2.071 & $1.150-6.368$ & 0.023 & $\mathrm{R}$ & 0.000 & 86.1 \\
\hline & Chinese & 2 & 14.66 & $6.729-31.95$ & $<1.0 \times 10^{-8}$ & $\mathrm{~F}$ & 0.613 & 0 \\
\hline & Italian & 3 & 1.535 & $0.735-3.206$ & 0.254 & $\mathrm{R}$ & 0.036 & 69.9 \\
\hline & Female & 2 & 1.953 & $0.957-3.985$ & 0.066 & $\mathrm{~F}$ & 0.294 & 9.28 \\
\hline & Male & 2 & 7.887 & $0.240-259.1$ & 0.246 & $\mathrm{R}$ & 0.049 & 74.2 \\
\hline
\end{tabular}

ESR1, estrogen receptor 1; $\mathrm{F}$, fixed model; $\mathrm{R}$, random model.

\section{Heterogeneity, meta-regression, sensitivity analysis, and publication bias}

Inter-study heterogeneity was observed in meta-analyses of the ESR1 PvuII and XbaI polymorphisms and $\mathrm{PP} / \mathrm{XX}$ haplotypes (Tables 2 and 3). Meta-regression showed that sample size $(P=0.013)$, but not ethnicity $(P=0.534)$, and publication year $(P=0.162)$ had a significant impact on the heterogeneity in the ESR1 PvuII polymorphism. Sensitivity analysis showed that no individual study significantly affected the pooled OR. Egger's regression test showed evidence of publication bias in the meta-analyses of the ESRI PvuII and XbaI polymorphisms (Egger's regression test $\mathrm{P}$ values $=0.085,0.017$ ). However, the adjusted ORs by the trim and fill technique were not significantly changed.

\section{DISCUSSION}

In the present study, we conducted a country- and gender-specific meta-analyses of the associations between the ESR1 PvuII and XbaI polymorphisms and AD susceptibility. We found an association between the ESR1 PvuII polymorphism and AD susceptibility in males, and an association between AD risk and the ESRI XbaI polymorphism in the Japanese population. We also detected an association between the PP/XX haplotype of the ESRI PvuII and $X b a \mathrm{I}$ polymorphisms and $\mathrm{AD}$ susceptibility in the Chinese population.

ESR1 polymorphisms may have a functional significance. Maruyama et al. (2000) found that ESRI PvuII and XbaI polymorphisms showed a weak enhancer activity, where the activity was higher in the $x$ allele than in the $X$ allele. Herrington et al. (2002) suggested that the $P$ allele underlines a functional myb transcription factor-binding site that could alter the transcription, stability, or structure of the ESR1 transcript and the subsequent ESR1 protein. 
There is also a possibility that ESRI PvuII and $X b a \mathrm{I}$ polymorphisms may be in LD with disease susceptibility genes.

The difference in the associations across countries, even within the same ethnic group remains unexplained. This could be due to unknown environmental factors and not ethnicity but country-related genetic factors. It is also unclear why a weak association of these polymorphisms and $\mathrm{AD}$ was observed in men in particular, in the different populations analyzed. This could be due to different levels of production and the effects of estrogen among men and women. However, we cannot rule out the possibility of false-positive type I error, due to the small number of studies included in this meta-analysis. Therefore, further studies with a large sample size are recommended.

Our study has several limitations. First, heterogeneity and confounding factors might have distorted the analysis. Second, it would have been interesting to evaluate the association between ESR1 polymorphisms and clinical features of $\mathrm{AD}$, but this was not possible because of the limited data.

In conclusion, this meta-analysis shows that the ESR1 PvuII polymorphism is associated with $\mathrm{AD}$ susceptibility in males, and that ESRI XbaI polymorphism is associated with $\mathrm{AD}$ risk in the Japanese population. In addition, the PP/XX haplotype is associated with $\mathrm{AD}$ susceptibility in the Chinese population. These data support that the ESRI PvuII and XbaI polymorphisms play a role in susceptibility to AD.

\section{Conflicts of interest}

The authors declare no conflict of interest.

\section{ACKNOWLEDGMENTS}

Research supported by a Korea University Grant.

\section{REFERENCES}

Bachman DL, Wolf PA, Linn R, Knoefel JE, et al. (1992). Prevalence of dementia and probable senile dementia of the Alzheimer type in the Framingham Study. Neurology 42: 115-119.

Boada M, Antunez C, López-Arrieta J, Caruz A, et al. (2012a). Estrogen receptor alpha gene variants are associated with Alzheimer's disease. Neurobiol. Aging 33: 198 e 115-124.

Boada M, Antunez C, Lopez-Arrieta J, Caruz A, et al. (2012b). Estrogen receptor alpha gene variants are associated with Alzheimer's disease. Neurobiol. Aging 33: 198 e 115-124.

Boccardi M, Scassellati C, Ghidoni R, Testa C, et al. (2008). Effect of the Xbal polymorphism of estrogen receptor alpha on postmenopausal gray matter. Neurosci. lett. 434: 304-309.

den Heijer T, Schuit SC, Pols HA, van Meurs JB, et al. (2004). Variations in estrogen receptor alpha gene and risk of dementia, and brain volumes on MRI. Mol. Psychiatry 9: 1129-1135.

Brandi ML, Becherini L, Gennari L, Racchi M, et al. (1999). Association of the estrogen receptor alpha gene polymorphisms with sporadic Alzheimer's disease. Biochem. Biophys. Res. Comm. 265: 335-338.

Corbo RM, Gambina G, Ruggeri M and Scacchi R (2006). Association of estrogen receptor alpha (ESR1) PvuII and XbaI polymorphisms with sporadic Alzheimer's disease and their effect on apolipoprotein E concentrations. Dement. Geriatr. Cogn. 22: 67-72.

Deng J, Shen C, Wang Y, Zhang M, et al. (2013). Association between the polymorphism of estrogen receptor alpha and Alzheimer's disease in Chinese population. Clin. Lab. 59: 741-746.

DerSimonian R and Laird N (1986). Meta-analysis in clinical trials. Control Clin. Trials 7: 177-188.

Dresner-Pollak R, Kinnar T, Friedlander Y, Sharon N, et al. (2009). Estrogen receptor beta gene variant is associated with 
vascular dementia in elderly women. Genet. Test. Mol. Biomarkers 13: 339-342.

Goumidi L, Dahlman-Wright K, Tapia-Paez I, Matsson H, et al. (2011). Study of estrogen receptor-alpha and receptorbeta gene polymorphisms on Alzheimer's disease. J. Alzheimers Dis. JAD 26: 431-439.

Herrington DM, Howard TD, Brosnihan KB, McDonnell DP, et al. (2002). Common estrogen receptor polymorphism augments effects of hormone replacement therapy on E-selectin but not C-reactive protein. Circulation 105: 1879-1882.

Higgins JP and Thompson SG (2002). Quantifying heterogeneity in a meta-analysis. Stat Med 21: 1539-1558.

Isoe-Wada K, Maeda M, Yong J, Adachi Y, et al. (1999). Positive association between an estrogen receptor gene polymorphism and Parkinson's disease with dementia. Eur. J. Neurol. 6: 431-435.

Ji Y, Urakami K, Wada-Isoe K, Adachi Y, et al. (2000). Estrogen receptor gene polymorphisms in patients with Alzheimer's disease, vascular dementia and alcohol-associated dementia. Dement. Geriatr. Cogn. Disord. 11: 119-122.

Lambert JC, Harris JM, Mann D, Lemmon H, et al. (2001). Are the estrogen receptors involved in Alzheimer's disease? Neurosci. Lett. 306: 193-197.

Lee YH and Nath SK (2005). Systemic lupus erythematosus susceptibility loci defined by genome scan meta-analysis. Hum. Genet. 118: 434-443.

Lee YH, Witte T, Momot T, Schmidt RE, et al. (2005). The mannose-binding lectin gene polymorphisms and systemic lupus erythematosus: two case-control studies and a meta-analysis. Arthritis Rheum. 52: 3966-3974.

Lee YH, Harley JB and Nath SK (2006). Meta-analysis of TNF-alpha promoter -308 A/G polymorphism and SLE susceptibility. Eur. J. Hum. Genet. 14: 364-371.

Lin GF, Ma QW, Zhang DS, Zha YL, et al. (2003). Polymorphism of alpha-estrogen receptor and aryl hydrocarbon receptor genes in dementia patients in Shanghai suburb. Acta pharmacol. Sin. 24: 651-656.

Ma SL, Tang NL, Tam CW, Lui VW, et al. (2009). Polymorphisms of the estrogen receptor alpha (ESR1) gene and the risk of Alzheimer's disease in a southern Chinese community. Int. Psychogeriatr. / IPA 21: 977-986.

Maruyama H, Toji H, Harrington CR, Sasaki K, et al. (2000). Lack of an association of estrogen receptor alpha gene polymorphisms and transcriptional activity with Alzheimer disease. Arch. Neurol. 57: 236-240.

Monastero R, Cefalù AB, Camarda C, Noto D, et al. (2006). Association of estrogen receptor alpha gene with Alzheimer's disease: a case-control study. J. Alzheimers Dis. JAD 9: 273-278.

Pirskanen M, Hiltunen M, Mannermaa A, Helisalmi S, et al. (2005). Estrogen receptor beta gene variants are associated with increased risk of Alzheimer's disease in women. Eur. J. Hum. Genet. 13: 1000-1006.

Porrello E, Monti MC, Sinforiani E, Cairati M, et al. (2006). Estrogen receptor alpha and APOEepsilon4 polymorphisms interact to increase risk for sporadic AD in Italian females. Eur. J. Neurol. 13: 639-644.

Szego EM, Csorba A, Janáky T, Kékesi KA, et al. (2011). Effects of estrogen on beta-amyloid-induced cholinergic cell death in the nucleus basalis magnocellularis. Neuroendocrinology 93: 90-105.

Usui C, Shibata N, Ohnuma T, Higashi S, et al. (2006). No genetic association between the myeloperoxidase gene -463 polymorphism and estrogen receptor-alpha gene polymorphisms and Japanese sporadic Alzheimer's disease. Dement. Geriatric Cog. Disorders 21: 296-299.

Xing Y, Jia JP, Ji XJ and Tian T (2013). Estrogen associated gene polymorphisms and their interactions in the progress of Alzheimer's disease. Prog. Neurobiol. 111C: 53-74. 